\title{
Haematological variables of Santa Ines and Ile de France suckling lambs: Influence of Haemonchus contortus infection ${ }^{1}$
}

\author{
José Gabriel G. Lins ${ }^{2,3 *}$ (D), Fabiana A. Almeida ${ }^{3}$ and Alessandro F.T. Amarante ${ }^{3}$
}

\begin{abstract}
Lins J.G.G., Almeida F.A. \& Amarante A.F.T. 2020. Haematological variables of Santa Ines and Ile de France suckling lambs: influence of Haemonchus contortus infection. Pesquisa Veterinária Brasileira 40(11):882-891. Graduate Program in Veterinary Medicine, Faculdade de Medicina Veterinária e Zootecnia, Universidade Estadual Paulista "Júlio de Mesquita Filho", Rua Prof. Doutor Walter Mauricio Correa s/n, Botucatu, SP 18618681, Brazil. E-mail: josegabriel_lins@hotmail.com

Haemonchus contortus is the major gastrointestinal parasite of sheep raised in tropical and subtropical areas worldwide. This trial aimed to evaluate the influence of $H$. contortus infection on the bone marrow response of Santa Ines (SI) and Ile de France (IF) suckling lambs experimentally infected with $H$. contortus. Fourteen SI lambs and 12 IF lambs were randomized in four groups: infected SI $(n=8)$, non-infected SI $(n=6)$, infected IF $(n=8)$ and non-infected IF $(n=4)$. Lambs of infected groups were submitted to 27 infections, conducted every two days, from 14 to 68 days of age, and each lamb received a total of $5400 \mathrm{H}$. contortus infective larvae (L3). Ten blood samples were obtained during the experimental period to be used for erythrocyte and leukocyte counts, packed cell volume and total plasma protein estimation. Additionally, it was carried out a differential leukocyte count. Lambs from control groups did not shed eggs in faeces all over the experiment, while infected Santa Ines and Ile de France lambs presented means of 2963 EPG (Eggs Per Gram of faeces) and 8175 EPG in the last sampling $(P<0.05)$, respectively. Infected Santa Ines lambs had an increase in eosinophil release, however differences $(\mathrm{P}<0.05)$ on circulation number in comparison with infected Ile de France lambs were identified only in the last sampling (54 days post first infection). The mild H. cortortus infection did not produce significant changes in the blood variables of the Ile de France and Santa Ines suckling lambs.
\end{abstract}

INDEX TERMS: Haemathology, sheep, Santa Ines, Ile de France, lamb, Haemonchus contortus, infection, Ovis aries, gastrointestinal nematodes, eosinophils.

\begin{abstract}
RESUMO.- [Variáveis hematológicas de cordeiros lactentes Santa Inês e Ile de France: influência da infecção por Haemonchus contortus.] Haemonchus contortus é o principal parasita gastrintestinal de ovinos criados em áreas tropicais e subtropicais. Este estudo objetivou avaliar a influência da infecção por $H$. contortus na resposta medular de cordeiros lactentes das raças Santa Inês (SI) e Ile de France (IF) infectados

\footnotetext{
${ }^{1}$ Received on July 5, 2020.

Accepted for publication on July 17, 2020.

${ }^{2}$ Graduate Program in Veterinary Medicine, Faculdade de Medicina Veterinária e Zootecnia (FMVZ), Universidade Estadual Paulista "Júlio de Mesquita Filho" (Unesp), Rua Prof. Doutor Walter Mauricio Correa s/n, Botucatu, SP 18618-681, Brazil. *Corresponding author: josegabriel_lins@hotmail.com

${ }^{3}$ Departamento de Bioestatística, Biologia Vegetal, Parasitologia e Zoologia, Instituto de Biociências, Universidade Estadual Paulista "Júlio de Mesquita Filho" (Unesp), Botucatu, SP 18618-000, Brazil.
}

experimentalmente com H. contortus. Quatorze cordeiros SI e 12 cordeiros IF, foram distribuídos em quatro grupos: SI infectado (n=8), SI não infectado $(n=6)$, IF infectado $(n=8)$ e IF não infectado $(n=4)$. Cordeiros dos grupos infectados foram submetidos a 27 infecções, realizadas a cada dois dias, do $14^{\circ}$ até $68^{\circ}$ dia de vida, com um total de 5400 larvas infectantes (L3) de H. contortus por animal. Foram realizadas 10 coletas de sangue ao longo do período experimental para determinação do volume globular, proteínas plasmáticas totais, contagem de eritrócitos e leucócitos, além do diferencial de leucócitos. Cordeiros controles não infectados não eliminaram ovos nas fezes durante todo o experimento, enquanto que os cordeiros Santa Ines e Ile de France infectados apresentaram em média 2963 ovos por grama de fezes (OPG) e 8175 OPG na última coleta $(\mathrm{P}<0,05)$, respectivamente. Cordeiros Santa Inês infectados tiveram um aumento na produção de eosinófilos, 
mas diferenças $(\mathrm{P}<0.05)$ no número de eosinófilos sanguíneos em comparação a cordeiros Ile de France infectados foram detectadas somente na última coleta (54 dias após a primeira infecção). A infecção leve por $H$. cortortus não induziu alterações significativas nas variáveis sanguíneas de cordeiros lactentes Ile de France e Santa Inês.

TERMOS DE INDEXAÇÃO: Hematologia, ovino, Santa Inês, Ile de France, cordeiro, Haemonchus contortus, infecção, Ovis aries, nematódeos gastrintestinais, eosinófilos.

\section{INTRODUCTION}

Occurrence of parasitic diseases in sheep is common, being an important cause of mortality, especially in young animals (Amarante \& Amarante 2016). Haemonchus contortus is an abomasal blood-sucking nematode considered one of the major gastrointestinal parasite of small ruminants in tropical and subtropical areas worldwide (Amarante 2014), mainly due its high pathogenicity and multiple resistance to anthelmintics (Almeida et al. 2010).

Haematological variables are sensitive indicators for the degree of parasitic infection severity, and animals presenting variables within the reference interval tend to have a better performance than those presenting any parasitic infection (Kumar et al. 2015). Heavy $H$. contortus infections might cause reduction in packed cell volume, and also in the total plasma protein concentration (Amarante et al. 2004), resulting consequently in the development of anaemia, hypoproteinemia and subsequent death even before the pre-patent period of the infection (Emery et al. 2016).

According to Weiss \& Wardrop (2010), the degree of anaemia varies according to age and resistance of infected hosts. Animal immunity plays an important role in resistance to worms, and individual factors such as age, breed and physiological condition regulate host response against parasites (Amarante 2014). Differences between sheep breeds regarding their susceptibility to infections by gastrointestinal nematodes have been reported (Amarante et al. 2004, Shakya et al. 2009). This is the case of Santa Ines sheep, a native breed from northeastern Brazil, considered more resistant to H. contortus infection when compared to Ile de France sheep (Amarante et al. 2004, Rocha et al. 2005, Amarante et al. 2009). Santa Ines lambs, after weaning, showed a higher resistance against $H$. contortus infection when compared to lambs of European breeds (Amarante et al. 2004, Albuquerque et al. 2019).

There are few studies that evaluated the immune response of suckling lambs against parasitic infections by gastrointestinal nematodes. Rocha et al. (2005) observed that Santa Ines lambs had higher eosinophil counts than Ile de France suckling lambs. Bahirathan et al. (1996) observed that Gulf Coast Native lambs had the ability to limit $H$. contortus infections by eliminating parasites, while Suffolk lambs developed haemonchosis and required anthelmintic treatment.

As haematological studies in suckling lambs infected with gastrointestinal nematodes in the first few days of life are limited, we carried out an evaluation on the haematological variables to assess the influence of $H$. contortus experimental infection on the bone marrow response of Santa Ines and Ile de France suckling lambs.

\section{MATERIALS AND METHODS}

Study area. The trial was conducted in the experimental area of the "Departamento de Bioestatística, Biologia Vegetal, Parasitologia e Zoologia", Instituto de Biociências, Universidade Estadual Paulista "Júlio de Mesquita Filho" (Unesp), Botucatu/SP, Brazil. All the procedures involving animals in this study were conducted in accordance with the local Ethics Committee on Animal Use (protocol number 0118/2018, FMVZ-Unesp).

Production of infective larvae (L3) of Haemonchus contortus. An $H$. contortus isolate susceptible to anthelmintics (Echevarria et al. 1991) stored in liquid nitrogen, and maintained at the "Departamento de Bioestatística, Biologia Vegetal, Parasitologia e Zoologia" of IBB, was thawed to infect two donor lambs. The worm-free status of donors was confirmed by several faecal examinations. Each lamb was infected orally with $5500 \mathrm{H}$. contortus L3 in a single dose.

After pre-patent period of the parasite, donor lambs faeces were collected individually into collecting plastic bags twice a day, and then, each sample was used to perform faecal cultures, according to Ueno \& Gonçalves (1998), for the production of L3 that was used in the infection of the experimental lambs.

Study design. Ile de France and Santa Ines pregnant ewes in the last third of gestation were acquired from two farms located in the rural area of São Manuel and Pardinho, respectively, both of them in São Paulo state, Brazil. After the naïve lambs were born, each ewe and its respective lamb were allocated into individual concrete floor pens with feeders and water fountains. Each pen was cleaned daily with water using a high-pressure pump in order to prevent undesired parasitic infections.

Fourteen Santa Ines lambs and 12 Ile de France lambs were randomized in four groups: infected Santa Ines ( $n=8)$, non-infected Santa Ines $(n=6)$, infected Ile de France $(n=8)$ and non-infected Ile de France $(n=4)$. The criterion for distribution of the lambs into the groups was the order of birth. The first male or female born of each breed was allocated in the infected group; the second female or male born was allotted in the control group, and then consecutively until all groups were composed. It was used a $2 \times 2$ factorial design, with two infection status (Infected and Control) and two sheep breeds (Santa Ines and Ile de France).

Food and sanitary management. Ewes and their lambs were fed with Cynodon spp. hay, free of nematode-infective larvae, and received a daily dietary supplement with $18 \%$ of crude protein (CP) (Nutrição Animal Coopermota ${ }^{\circledR}$ ). Additionally, the animals had free access to tap water and mineral salt (OvinoFós Tortuga ${ }^{\circledR}$ ).

Experimental infections. Naïve lambs of the infected groups were infected, from 14 days until 66 days old, with $H$. contortus L3 through a serial infection protocol with a progressive increase in L3 numbers. Experimental infections were carried out each two days, being divided into three steps, differing in the number of L3 administered at each stage. In total, it was conducted 27 direct artificial infections (Fig.1). Infective larvae were administered directly into the lamb oral cavities. At the end of the infections (at 66 days of life), each lamb had received a total of $5400 \mathrm{L3}$. Control groups were kept worm-free during the whole experiment.

Data collection. Blood samples were obtained by jugular vein puncture into plain tubes containing anticoagulant (Vacutainer ${ }^{\circledR} \mathrm{K} 2$ EDTA 7.2mg, BD, Brazil). Haematological exams performed were packed cell volume (PCV), total plasma protein estimation (TPP), red blood cell count (RBC), white blood cell count (WBC) and blood smear for differential leukocyte count (Jain 1986, Weiss \& Wardrop 2010, Weiser 2012). 
Table 1. Packed cell volume in percentage (\%) means ( \pm standard error) of Ile de France and Santa Ines suckling lambs experimentally infected with Haemonchus contortus and non-infected control

\begin{tabular}{|c|c|c|c|c|c|c|}
\hline \multirow{2}{*}{ DPI } & \multicolumn{2}{|c|}{ Ile de France } & \multicolumn{2}{|c|}{ Santa Ines } & \multicolumn{2}{|c|}{ Effects ( $P$-value $)$} \\
\hline & Infected $(\mathrm{n}=8)$ & Control $(n=4)$ & Infected $(n=8)$ & Control $(n=6)$ & Breed & Infection status \\
\hline 0 & $36( \pm 1.11)$ a & $35( \pm 1.03) \mathrm{ab}$ & $30( \pm 1.87) \mathrm{ab}$ & $29( \pm 1.94) b$ & 0.0071 & ns \\
\hline 6 & $34( \pm 1.12)$ a & $34( \pm 0.33) \mathrm{a}$ & $28( \pm 1.87) b c$ & $25( \pm 1.61) \mathrm{c}$ & 0.0002 & ns \\
\hline 12 & $32( \pm 1.05) \mathrm{a}$ & $34( \pm 1.49) \mathrm{ab}$ & $27( \pm 1.82) \mathrm{bc}$ & $25( \pm 1.15) \mathrm{c}$ & 0.0007 & ns \\
\hline 18 & $32( \pm 1.32) \mathrm{ab}$ & $35( \pm 0.96) b$ & $27( \pm 1.1) \mathrm{ac}$ & $26( \pm 1.08) c$ & 0.0002 & ns \\
\hline 24 & $32( \pm 1.03)$ & $37( \pm 0.25)$ & $30( \pm 1.85)$ & $30( \pm 1.33)$ & 0.0153 & ns \\
\hline 30 & $33( \pm 0.74) \mathrm{ab}$ & $36( \pm 0.91) \mathrm{a}$ & $31( \pm 1.16) b$ & $32( \pm 1.33) \mathrm{ab}$ & 0.01 & ns \\
\hline 36 & $33( \pm 0.53)$ & $36( \pm 2.29)$ & $33( \pm 1.0)$ & $35( \pm 1.77)$ & ns & ns \\
\hline 42 & $34( \pm 0.81)$ & $38( \pm 1.35)$ & $34( \pm 0.85)$ & $36( \pm 2.19)$ & ns & 0.0293 \\
\hline 48 & $34( \pm 1.32)$ & $37( \pm 2.04)$ & $34( \pm 1.13)$ & $35( \pm 2.29)$ & ns & ns \\
\hline 54 & $36( \pm 1.13)$ & $39( \pm 1.03)$ & $35( \pm 1.43)$ & $38( \pm 2.25)$ & ns & ns \\
\hline
\end{tabular}

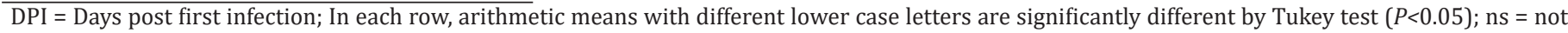
significant $(P>0.05)$; There was no significant interaction between breed $\mathrm{x}$ infection status $(P>0.05)$.

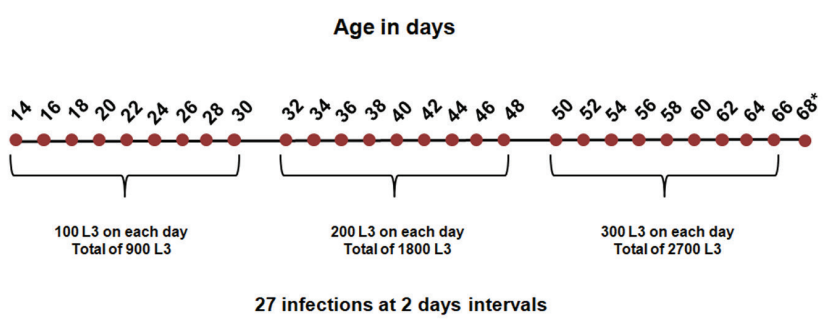

Fig.1. Timeline with the protocol of the experimental infections (27 at two days intervals) of the lambs with 5400 infective larvae (L3) of Haemonchus contortus. The last sampling was conducted at 68 days of age (asterisk).

Packed cell volume was determined by microhematocrit centrifugation (5 min/15000g, Fanem 211 microhematocrit centrifuge), and TPP concentrations were estimated using a hand held refractometer (Refractometer SPR-N, Atago).

Red blood cell count was performed manually in a Neubauer chamber using a 1:201 of blood $(20 \mu \mathrm{L}$ of blood and $4000 \mu \mathrm{L}$ of saline solution $0.9 \% \mathrm{NaCl}$. The dilution was mixed continuously for two minutes by mechanical shaker. White blood cell count was performed manually in a Neubauer chamber using a 1:20 dilution of blood ( $20 \mu \mathrm{L}$ of blood and $380 \mu \mathrm{L}$ of Turk`s solution) (Jain 1986).

For differential leukocyte count, blood smear was stained using a Diff Quick stain (Panótico Rápido, Laborclin/PR, Brazil). Additionally, 100 leukocytes were differentiated and results were presented in absolute values (Jain 1986).

Faecal samples were collected directly from the rectum of animals. Faecal egg counts determination was performed using a modified McMaster technique in which each worm egg counted represented 100 eggs per gram (EPG) of faeces (Ueno \& Gonçalves 1998).

Finally, it was conducted 10 sampling data collection in total, every six days, from 14 to 68 days old.

Statistical analysis. Data were evaluated in a normality test (Shapiro-Wilk test), and only PCV and TPP had normal distribution. Cell count results were non-normally distributed, and were transformed to $\log 10(x+1)$ prior to analysis. Furthermore, data were analyzed by analysis of variance through the General Linear Model (GLM) for variables determined at several time points, using the Statistical Analysis System, version 9.2 (SAS Institute, Inc., Cary/NC, USA). Means were compared by Tukey's test at a 5\% significance level, and only significant interactions were reported in the results. Means are presented in the results as the arithmetic means ( \pm standard error).
Table 2. Total plasma protein (TPP, g/dL) means $( \pm$ standard error) of Ile de France and Santa Ines suckling lambs experimentally infected with Haemonchus contortus and non-infected control

\begin{tabular}{ccccc}
\hline \multirow{2}{*}{ DPI } & \multicolumn{2}{c}{ Ile de France } & \multicolumn{2}{c}{ Santa Ines } \\
\cline { 2 - 5 } & Infected $(\mathrm{n}=8)$ & Control $(\mathrm{n}=4)$ & Infected $(\mathrm{n}=8)$ & Control $(\mathrm{n}=6)$ \\
\hline 0 & $5.6( \pm 0.12)$ & $5.9( \pm 0.24)$ & $5.8( \pm 0.19)$ & $6.1( \pm 0.34)$ \\
6 & $5.6( \pm 0.15)$ & $5.9( \pm 0.18)$ & $5.8( \pm 0.23)$ & $5.9( \pm 0.35)$ \\
12 & $5.6( \pm 0.17)$ & $5.8( \pm 0.13)$ & $5.7( \pm 0.14)$ & $5.7( \pm 0.37)$ \\
18 & $5.8( \pm 0.17)$ & $5.7( \pm 0.22)$ & $5.5( \pm 0.15)$ & $5.5( \pm 0.36)$ \\
24 & $5.6( \pm 0.20)$ & $5.9( \pm 0.13)$ & $5.5( \pm 0.19)$ & $5.6( \pm 0.29)$ \\
30 & $5.5( \pm 0.24)$ & $5.5( \pm 0.15)$ & $5.7( \pm 0.17)$ & $5.6( \pm 0.35)$ \\
36 & $5.7( \pm 0.29)$ & $5.9( \pm 0.05)$ & $5.5( \pm 0.18)$ & $5.7( \pm 0.32)$ \\
42 & $5.7( \pm 0.28)$ & $6.0( \pm 0.15)$ & $5.8( \pm 0.17)$ & $5.8( \pm 0.26)$ \\
48 & $5.8( \pm 0.24)$ & $6.0( \pm 0.14)$ & $5.9( \pm 0.22)$ & $5.9( \pm 0.26)$ \\
54 & $5.8( \pm 0.23)$ & $6.2( \pm 0.14)$ & $6.0( \pm 0.21)$ & $6.0( \pm 0.23)$
\end{tabular}

DPI = Days post first infection; There were no breed and infection status effects, and breed $\mathrm{x}$ infection status interaction on TPP values $(P>0.05)$.

\section{RESULTS}

Lambs from control groups did not shed egg in faeces all over the experiment, while infected Santa Ines and Ile de France lambs presented in average 2963 EPG and 8175 EPG in the last sampling $(P<0.05)$, respectively.

There was a time effect $(P<0.0001)$ on PCV values (Table 1$)$. Breed had a significant effect on PCV values from day 14 to 44 days of age, with Santa Ines lambs showing the lowest PCV values. In addition, there was an infection status effect $(P<0.05)$ at 56 days of age (42 days post first infection - DPI) with control lambs showing the highest PCV means.

Furthermore, there was time effect $(P=0.0153)$ on the TPP concentration (Table 2). Over the experiment, most of the TPP means remained below the reference interval for sheep.

In relation to $\mathrm{RBC}$ counting, it was noted a breed effect at $20,44,50,56,62$ and 68 days old $(6,30,36,42,48$ and 54 DPI, respectively), with Santa Ines lambs presenting the highest RBC means from 44 to 68 days of age. Red blood cell trend was similar in both breeds, and the counting increased slightly as the suckling lambs got older (Table 3). There was time effect $(P<0.0001)$ and a significant Time $\mathrm{x}$ Breed interaction $(P<0.0001)$ on the RBC counting with Santa Ines lambs showing higher means in the last five samplings. 
Infected Santa Ines lambs presented a higher number of RBC when compared to infected Ile de France lambs at $44(P=0.017)$ and $56(P=0.0346)$ days old (30 and 42 DPI, respectively). However RBC values of infected groups were within the reference interval.
White blood cell counts of infected and non-infected (control) groups are shown in Table 4. There was no significant difference among the groups all over the experiment. Breed influence $(P=0.0182)$ occurred only at 32 days old (18 DPI) when Ile de France lambs had the highest counts. All values

Table 3. Red blood cell (x 10\% $/ \mu \mathrm{L})$ means (minimum and maximum values) of Ile de France and Santa Ines suckling lambs experimentally infected with Haemonchus contortus and non-infected control

\begin{tabular}{|c|c|c|c|c|c|}
\hline \multirow{2}{*}{ DPI } & \multicolumn{2}{|c|}{ Ile de France } & \multicolumn{2}{|c|}{ Santa Ines } & \multirow{2}{*}{$\frac{\text { Effect }(P \text {-value })}{\text { Breed }}$} \\
\hline & Infected $(n=8)$ & Control (n=4) & Infected $(n=8)$ & Control $(n=6)$ & \\
\hline 0 & $\begin{array}{c}8.7(7.6-9.9) \\
\# 0.98( \pm 0.01)\end{array}$ & $\begin{array}{l}7.9(7.4-8.6) \\
0.95( \pm 0.01)\end{array}$ & $\begin{array}{c}8.0(5.8-9.82) \\
0.95( \pm 0.02)\end{array}$ & $\begin{array}{l}8.2(6.8-9.7) \\
0.96( \pm 0.02)\end{array}$ & ns \\
\hline 6 & $\begin{array}{c}9.5(8.2-11) \\
1.02( \pm 0.01) \mathrm{a}\end{array}$ & $\begin{array}{l}9.16(8.3-10.0) \\
0.98( \pm 0.03) \mathrm{ab}\end{array}$ & $\begin{array}{l}8.3(7.11-9.97) \\
0.97( \pm 0.02) a b\end{array}$ & $\begin{array}{c}7.8(6.8-9.0) \\
0.94( \pm 0.01) b\end{array}$ & 0.0173 \\
\hline 12 & $\begin{array}{c}9.4(8.4-11.3) \\
1.01( \pm 0.01)\end{array}$ & $\begin{array}{l}9.1(8.1-9.9) \\
1.0( \pm 0.02)\end{array}$ & $\begin{array}{c}8.8(7.63-10.5) \\
0.99( \pm 0.01)\end{array}$ & $\begin{array}{l}8.3(7.7-9.5) \\
0.97( \pm 0.01)\end{array}$ & ns \\
\hline 18 & $\begin{array}{c}9.4(7.3-10.4) \\
1.02( \pm 0.01)\end{array}$ & $\begin{array}{c}9.6(8.7-10.5) \\
1.03( \pm 0.02)\end{array}$ & $\begin{array}{c}9.4(8.25-10.2) \\
1.02( \pm 0.01)\end{array}$ & $\begin{array}{l}8.4(7.4-9.7) \\
0.97( \pm 0.02)\end{array}$ & ns \\
\hline 24 & $\begin{array}{c}9.1(6.9-10.6) \\
1.0( \pm 0.02)\end{array}$ & $\begin{array}{c}10.3(9.5-10.8) \\
1.05( \pm 0.01)\end{array}$ & $\begin{array}{c}9.8(9.1-12.6) \\
1.03( \pm 0.01)\end{array}$ & $\begin{array}{c}10.0(9.6-10.6) \\
1.04( \pm 0.006)\end{array}$ & ns \\
\hline 30 & $\begin{array}{l}9.3(7.5-10.6) \\
1.01( \pm 0.01) \mathrm{a}\end{array}$ & $\begin{array}{c}10.0(9.7-10.4) \\
1.04( \pm 0.007) \mathrm{ab}\end{array}$ & $\begin{array}{l}10.9(9.1-12.6) \\
1.07( \pm 0.02) \mathrm{b}\end{array}$ & $\begin{array}{l}11.1(9.4-12.6) \\
1.08( \pm 0.02) b\end{array}$ & 0.0074 \\
\hline 36 & $\begin{array}{l}9.6(7.3-10.6) \\
1.02( \pm 0.02) \mathrm{a}\end{array}$ & $\begin{array}{c}9.8(8.6-10.4) \\
1.03( \pm 0.02) a b\end{array}$ & $\begin{array}{c}11(8.7-14.1) \\
1.08( \pm 0.02) a b\end{array}$ & $\begin{array}{c}11.9(10.1-13.7) \\
1.11( \pm 0.02) \mathrm{b}\end{array}$ & 0.0052 \\
\hline 42 & $\begin{array}{c}9.4(5-12.5) \\
1.01( \pm 0.04) \mathrm{a}\end{array}$ & $\begin{array}{l}10.1(8.3-12.0) \\
1.04( \pm 0.03) \mathrm{ab}\end{array}$ & $\begin{array}{l}12.3(9.7-15.1) \\
1.12( \pm 0.02) \mathrm{b}\end{array}$ & $\begin{array}{l}12.1(8.9-14.0) \\
1.11( \pm 0.03) \mathrm{ab}\end{array}$ & 0.0094 \\
\hline 48 & $\begin{array}{c}10.2(8.7-13.0) \\
1.05( \pm 0.02)\end{array}$ & $\begin{array}{c}10.5(9.0-12.2) \\
1.06( \pm 0.02)\end{array}$ & $\begin{array}{c}12.0(10-14.5) \\
1.11( \pm 0.02)\end{array}$ & $\begin{array}{c}12.0(9.7-17.2) \\
1.11( \pm 0.03)\end{array}$ & 0.0329 \\
\hline 54 & $\begin{array}{c}10.3(8.4-14.4) \\
1.05( \pm 0.02) \mathrm{a} \\
\end{array}$ & $\begin{array}{c}11.7(10.4-12.4) \\
1.1( \pm 0.02) \mathrm{ab}\end{array}$ & $\begin{array}{l}12.9(9.9-15.7) \\
1.14( \pm 0.03) \mathrm{ab}\end{array}$ & $\begin{array}{c}13.8(10.3-16.2) \\
1.16( \pm 0.03) \mathrm{b}\end{array}$ & 0.0129 \\
\hline
\end{tabular}

DPI = Days post first infection; \# means ( \pm standard error) of data transformed using $\log (\mathrm{x}+1)$; In each row, arithmetic means with different lower case letters are significantly different by Tukey test $(P<0.05)$; ns $=$ not significant $(P>0.05)$; There were no infection status effects, and breed $\mathrm{x}$ infection status interaction on red blood cell counts $(P>0.05)$.

Table 4. White blood cell (cells/ $\mu \mathrm{L}$ ) means (minimum and maximum values) of Ile de France and Santa Ines suckling lambs experimentally infected with Haemonchus contortus and non-infected control

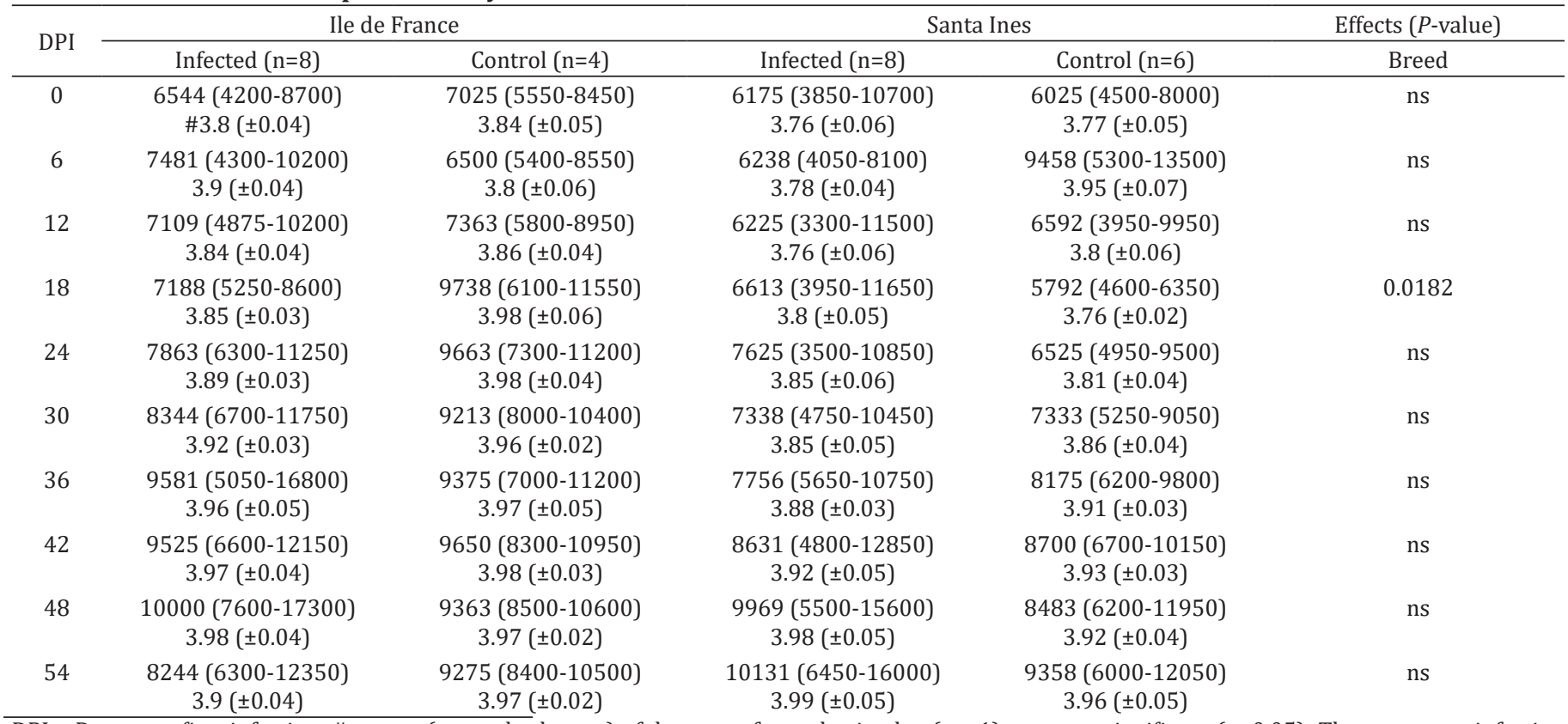

DPI = Days post first infection; \# means $( \pm$ standard error) of data transformed using log $(x+1)$; ns $=$ not significant $(p>0.05)$; There were no infection status effects, and breed $\mathrm{x}$ infection status interaction on white blood cell counts $(P>0.05)$. 
found for WBC were within the reference interval for the species. There was a significant time effect $(P<0.0001)$, Time $\mathrm{x}$ Breed $(P=0.0479)$ and Time $\mathrm{x}$ Breed $\mathrm{x}$ Infection status $(P=0.0326)$ interactions on the WBC counting.

Differential leukocyte counting is presented in absolute numbers. With regards to eosinophils means, there was a breed effect $(P=0.0037)$ on eosinophils counting at 20 days old (6 DPI), with a significant difference only between infected and non-infected Santa Ines lambs $(P=0.0037)$. A similar result was observed at 42 DPI (56 days old), when infected Santa Ines lambs had higher number of eosinophils than non-infected Santa Ines counterparts $(P=0.0242)$. There was a significant Breed $x$ Infection status interaction on eosinophils counting at 24, 36, 42 and 54 DPI (38, 50, 56 and 68 days old). Additionally, infected Santa Ines lambs had significantly higher number $(P=0.0256)$ of eosinophils at 68 days old, when compared to infected Ile de France lambs (Table 5).

There was an infection status effect on neutrophils counting $(P=0.0481)$ at 6 DPI (20 days old) (Table 6). Infected Santa Ines lambs had lower neutrophil count than non-infected

Table 5. Eosinophil (cells $/ \mu \mathrm{L}$ ) means (minimum and maximum values) of Ile de France and Santa Ines suckling lambs experimentally infected with Haemonchus contortus and non-infected control

\begin{tabular}{|c|c|c|c|c|c|c|c|}
\hline \multirow{2}{*}{ DPI } & \multicolumn{2}{|c|}{ Ile de France } & \multicolumn{2}{|c|}{ Santa Ines } & \multicolumn{3}{|c|}{ Effects ( $P$-value $)$} \\
\hline & Infected $(n=8)$ & Control $(n=4)$ & Infected $(\mathrm{n}=8)$ & Control $(n=6)$ & Breed & Infection status & Breed x Infection status \\
\hline 0 & $\begin{array}{l}398(49-959) \\
\# 2.42( \pm 0.16)\end{array}$ & $\begin{array}{c}355(111-761) \\
2.42( \pm 0.18)\end{array}$ & $\begin{array}{c}323(52-1071) \\
2.31( \pm 0.16)\end{array}$ & $\begin{array}{l}254(0-576) \\
1.71( \pm 0.54)\end{array}$ & ns & ns & ns \\
\hline 6 & $\begin{array}{l}492(61-918) \\
2.54( \pm 0.17) \mathrm{a}\end{array}$ & $\begin{array}{l}311(171-540) \\
2.55( \pm 0.15) a\end{array}$ & $\begin{array}{l}216(41-568) \\
2.18( \pm 0.14) \text { a }\end{array}$ & $\begin{array}{c}80(0-212) \\
1.1( \pm 0.49) \mathrm{b}\end{array}$ & 0.0037 & ns & ns \\
\hline 12 & $\begin{array}{l}286(0-918) \\
1.85( \pm 0.42)\end{array}$ & $\begin{array}{c}484(116-1074) \\
2.54( \pm 0.21)\end{array}$ & $\begin{array}{c}169(48-504) \\
2.11( \pm 0.12)\end{array}$ & $\begin{array}{l}199(0-624) \\
1.52( \pm 0.51)\end{array}$ & ns & ns & ns \\
\hline 18 & $\begin{array}{l}139(0-453) \\
1.82( \pm 0.28)\end{array}$ & $\begin{array}{c}423(217-941) \\
2.54( \pm 0.15)\end{array}$ & $\begin{array}{c}191(0-557) \\
1.95( \pm 0.3)\end{array}$ & $\begin{array}{c}139(46-315) \\
2.06( \pm 0.12)\end{array}$ & ns & ns & ns \\
\hline 24 & $\begin{array}{l}105(0-258) \\
1.16( \pm 0.44)\end{array}$ & $\begin{array}{c}469(91-1232) \\
2.48( \pm 0.24)\end{array}$ & $\begin{array}{c}247(70-508) \\
2.3( \pm 0.11)\end{array}$ & $\begin{array}{c}95(0-285) \\
1.39( \pm 0.45)\end{array}$ & ns & ns & 0.0064 \\
\hline 30 & $\begin{array}{c}220(0-668) \\
1.79( \pm 0.4)\end{array}$ & $\begin{array}{c}474(80-1128) \\
2.5( \pm 0.24)\end{array}$ & $\begin{array}{c}433(95-1012) \\
2.52(0.12)\end{array}$ & $\begin{array}{c}223(67-496) \\
2.25( \pm 0.14)\end{array}$ & ns & ns & ns \\
\hline 36 & $\begin{array}{l}133(0-344) \\
1.62( \pm 0.37)\end{array}$ & $\begin{array}{c}405(87-959) \\
2.46( \pm 0.22)\end{array}$ & $\begin{array}{c}452(170-1656) \\
2.52( \pm 0.11)\end{array}$ & $\begin{array}{l}198(0-496) \\
1.63( \pm 0.52)\end{array}$ & ns & ns & 0.0240 \\
\hline 42 & $\begin{array}{c}210(0-495) \\
2.0( \pm 0.30) \mathrm{ab}\end{array}$ & $\begin{array}{c}350(83-657) \\
2.37( \pm 0.24) a b\end{array}$ & $\begin{array}{c}443(120-994) \\
2.57( \pm 0.10) \mathrm{a}\end{array}$ & $\begin{array}{c}108(0-335) \\
1.14( \pm 0.52) b\end{array}$ & ns & ns & 0.0126 \\
\hline 48 & $\begin{array}{c}244(79-519) \\
2.31( \pm 0.1)\end{array}$ & $\begin{array}{c}225(106-425) \\
2.30( \pm 0.12)\end{array}$ & $\begin{array}{c}500(96-1212) \\
2.59( \pm 0.13)\end{array}$ & $\begin{array}{c}228(91-372) \\
2.31( \pm 0.1)\end{array}$ & ns & ns & ns \\
\hline 54 & $\begin{array}{c}138(0-247) \\
1.69( \pm 0.37) \mathrm{a}\end{array}$ & $\begin{array}{l}458(281-672) \\
2.63( \pm 0.08) a b\end{array}$ & $\begin{array}{l}508(160-867) \\
2.63( \pm 0.10) \mathrm{b}\end{array}$ & $\begin{array}{c}148(97-279) \\
2.14( \pm 0.07) a b\end{array}$ & ns & ns & 0.0095 \\
\hline
\end{tabular}

DPI = Days post first infection; \# means $( \pm$ standard error) of data transformed using log $(\mathrm{x}+1)$; In each row, arithmetic means with different lower case letters are significantly different by Tukey test $(P<0.05)$; ns = not significant $(P>0.05)$.

Table 6. Neutrophil (cells/ $\mu \mathrm{L}$ ) means (minimum and maximum values) of Ile de France and Santa Ines suckling lambs experimentally infected with Haemonchus contortus and non-infected control

\begin{tabular}{|c|c|c|c|c|c|}
\hline \multirow{2}{*}{ DPI } & \multicolumn{2}{|c|}{ Ile de France } & \multicolumn{2}{|c|}{ Santa Ines } & \multirow{2}{*}{$\frac{\text { Effects ( } P \text {-value })}{\text { Infection status }}$} \\
\hline & Infected $(n=8)$ & Control $(n=4)$ & Infected $(n=8)$ & Control $(n=6)$ & \\
\hline \multirow[t]{2}{*}{0} & $2769(1308-4300)$ & 3337 (2498-4452) & 3055 (1519-5243) & $3306(1786-5076)$ & ns \\
\hline & $\# 3.41( \pm 0.06)$ & $3.51( \pm 0.06)$ & $3.44( \pm 0.07)$ & $3.49( \pm 0.07)$ & \\
\hline \multirow[t]{2}{*}{6} & $2584(1333-3672)$ & $3311(1404-5643)$ & $2617(1134-4350)$ & $6396(2096-10004)$ & 0.0481 \\
\hline & $3.39( \pm 0.06) \mathrm{a}$ & $3.45( \pm 0.3) \mathrm{ab}$ & $3.39( \pm 0.06) \mathrm{a}$ & $3.73( \pm 0.12) b$ & \\
\hline \multirow[t]{2}{*}{12} & $2334(975-3978)$ & $2797(2280-3491)$ & $2869(825-6670)$ & $3606(2054-6048)$ & ns \\
\hline & $3.32( \pm 0.08)$ & $3.44( \pm 0.17)$ & $3.39( \pm 0.09)$ & $3.51( \pm 0.09)$ & \\
\hline \multirow[t]{2}{*}{18} & $2385(1304-4043)$ & $4919(2074-8138)$ & $2480(672-6058)$ & $2332(1260-3422)$ & ns \\
\hline & $3.35( \pm 0.06)$ & $3.63( \pm 0.04)$ & $3.31( \pm 0.1)$ & $3.33( \pm 0.08)$ & \\
\hline \multirow[t]{2}{*}{24} & 2936 (1935-5063) & 3815 (2628-5159) & $2981(945-4557)$ & $2519(1040-4275)$ & ns \\
\hline & $3.45( \pm 0.05)$ & $3.57( \pm 0.13)$ & $3.42( \pm 0.09)$ & $3.35( \pm 0.09)$ & \\
\hline \multirow[t]{2}{*}{30} & $2249(1192-3525)$ & $3375(3008-3620)$ & $2921(1140-5225)$ & $3108(1628-4446)$ & ns \\
\hline & $3.32( \pm 0.06)$ & $3.53( \pm 0.06)$ & $3.42( \pm 0.07)$ & $3.47( \pm 0.07)$ & \\
\hline \multirow[t]{2}{*}{36} & 3985 (1414-12432) & $2819(2450-3136)$ & $2858(1541-4623)$ & $3671(2146-6174)$ & ns \\
\hline & $3.5( \pm 0.1)$ & $3.45( \pm 0.02)$ & $3.44( \pm 0.05)$ & $3.54( \pm 0.06)$ & \\
\hline \multirow[t]{2}{*}{42} & 3159 (1188-5429) & $3602(2883-5037)$ & $3570(1440-6570)$ & 4167 (3082-5887) & ns \\
\hline & $3.45( \pm 0.08)$ & $3.55( \pm 0.05)$ & $3.5( \pm 0.07)$ & $3.61( \pm 0.04)$ & \\
\hline \multirow[t]{2}{*}{48} & 3434 (1238-9688) & $3548(2635-4346)$ & 3927 (1540-9204) & $4661(2604-7768)$ & ns \\
\hline & $3.44( \pm 0.1)$ & $3.54( \pm 0.06)$ & $3.53( \pm 0.08)$ & $3.64( \pm 0.07)$ & \\
\hline \multirow[t]{2}{*}{54} & $2667(704-4940)$ & $3172(2415-4602)$ & $3762(1812-9760)$ & $4718(2280-8556)$ & ns \\
\hline & $3.35( \pm 0.1)$ & $3.49( \pm 0.06)$ & $3.51( \pm 0.08)$ & $3.64( \pm 0.08)$ & \\
\hline
\end{tabular}

DPI = Days post first infection; \# means ( \pm standard error) of data transformed using log ( $\mathrm{x}+1$ ); In each row, arithmetic means with different lower case letters are significantly different by Tukey test $(P<0.05)$; ns = not significant $(P>0.05)$; There were not breed effect and breed $\mathrm{x}$ infection status interaction on Neutrophil count $(P>0.05)$. 
Santa Ines $(P=0.0376)$. There was a significant Time $\mathrm{x}$ Breed interaction $(P=0.0125)$ on the neutrophil counting.

Regarding to basophils counting (Table 7), infected Santa Ines lambs had higher values when compared to infected Ile de France, furthermore there was a significant difference between infected groups only at 6 DPI $(P=0.0001)$.
It was observed a time effect $(P<0.0001)$ on the lymphocytes number (Table 8). Infected Ile de France lambs had higher lymphocytes numbers than infected Santa Ines at 12 and 30 DPI (26 and 44 days old) ( $P=0.0117$ and $P=0.0043$, respectively). There was breed effect on lymphocyte counting at $12,30,36$, 42 and 48 DPI (26, 44, 50, 56 and 62 days old), and infection

Table 7. Basophil (cells/ $\mu \mathrm{L}$ ) means (minimum and maximum values) of Ile de France and Santa Ines suckling lambs experimentally infected with Haemonchus contortus and non-infected control

\begin{tabular}{|c|c|c|c|c|c|}
\hline \multirow{2}{*}{ DPI } & \multicolumn{2}{|c|}{ Ile de France } & \multicolumn{2}{|c|}{ Santa Ines } & \multirow{2}{*}{$\frac{\text { Effects }(P \text {-value })}{\text { Breed }}$} \\
\hline & Infected $(\mathrm{n}=8)$ & Control $(n=4)$ & Infected $(\mathrm{n}=8)$ & Control $(n=6)$ & \\
\hline 0 & $\begin{array}{c}49(0-137) \\
\# 0.99( \pm 0.38)\end{array}$ & $\begin{array}{l}77(0-168) \\
1.48( \pm 0.5)\end{array}$ & $\begin{array}{l}113(0-230) \\
1.81( \pm 0.27)\end{array}$ & $\begin{array}{c}82(0-144) \\
1.64( \pm 0.33)\end{array}$ & ns \\
\hline 6 & $\begin{array}{c}25(0-126) \\
0.5( \pm 0.33) \mathrm{a}\end{array}$ & $\begin{array}{c}37(0-56) \\
1.16( \pm 0.58) \mathrm{ab}\end{array}$ & $\begin{array}{l}198(52-355) \\
2.24( \pm 0.09) b\end{array}$ & $\begin{array}{l}144(64-368) \\
2.08( \pm 0.11) b\end{array}$ & 0.0001 \\
\hline 12 & $\begin{array}{c}71(0-146) \\
1.27( \pm 0.38)\end{array}$ & $\begin{array}{c}74(58-90) \\
1.87( \pm 0.04)\end{array}$ & $\begin{array}{l}103(0-220) \\
1.56( \pm 0.35)\end{array}$ & $\begin{array}{l}56(0-126) \\
1.27( \pm 0.4)\end{array}$ & ns \\
\hline 18 & $\begin{array}{c}39(0-125) \\
0.93( \pm 0.36)\end{array}$ & $\begin{array}{c}86(0-122) \\
1.54( \pm 0.52)\end{array}$ & $\begin{array}{c}102(0-375) \\
1.34( \pm 0.4)\end{array}$ & $\begin{array}{c}95(0-173) \\
1.69( \pm 0.34)\end{array}$ & ns \\
\hline 24 & $\begin{array}{c}80(0-210) \\
1.49( \pm 0.33)\end{array}$ & $\begin{array}{c}64(0-146) \\
1.05( \pm 0.61)\end{array}$ & $\begin{array}{c}178(0-305 \\
1.99( \pm 0.29)\end{array}$ & $\begin{array}{c}47(0-118) \\
0.98( \pm 0.44)\end{array}$ & ns \\
\hline 30 & $\begin{array}{c}45(0-118) \\
0.97( \pm 0.37)\end{array}$ & $\begin{array}{c}95(0-181) \\
1.56( \pm 0.52)\end{array}$ & $\begin{array}{c}95(0-244) \\
1.53( \pm 0.34)\end{array}$ & $\begin{array}{c}30(0-67) \\
0.89( \pm 0.39)\end{array}$ & ns \\
\hline 36 & $\begin{array}{c}65(0-157) \\
1.24( \pm 0.37)\end{array}$ & $\begin{array}{c}75(0-213) \\
1.07( \pm 0.62)\end{array}$ & $\begin{array}{l}139(0-335) \\
1.68( \pm 0.37)\end{array}$ & $\begin{array}{c}71(0-166) \\
1.34( \pm 0.43)\end{array}$ & ns \\
\hline 42 & $\begin{array}{c}69(0-122) \\
1.47( \pm 0.32)\end{array}$ & $\begin{array}{c}72(0-110) \\
1.48( \pm 0.49)\end{array}$ & $\begin{array}{c}113(0-257) \\
1.8( \pm 0.27)\end{array}$ & $\begin{array}{c}70(0-185) \\
1.07( \pm 0.48)\end{array}$ & ns \\
\hline 48 & $\begin{array}{c}77(0-210) \\
1.29( \pm 0.38)\end{array}$ & $\begin{array}{l}51(0-106) \\
1.0( \pm 0.58)\end{array}$ & $\begin{array}{l}125(0-312) \\
1.62( \pm 0.36)\end{array}$ & $\begin{array}{c}35(0-120) \\
0.67( \pm 0.43)\end{array}$ & ns \\
\hline 54 & $\begin{array}{c}63(0-247) \\
1.02( \pm 0.39)\end{array}$ & $\begin{array}{c}96(0-210) \\
1.55( \pm 0.52)\end{array}$ & $\begin{array}{l}111(0-269) \\
1.39( \pm 0.41)\end{array}$ & $\begin{array}{c}75(0-241) \\
1.07( \pm 0.48)\end{array}$ & ns \\
\hline
\end{tabular}

DPI = Days post first infection; \# means $( \pm$ standard error $)$ of data transformed using log $(x+1)$; In each row, arithmetic means with different lower case letters are significantly different by Tukey test $(P<0.05)$; ns = not significant $(P>0.05)$; There were no infection status effect and breed $\mathrm{x}$ infection status interaction on Basophil count $(P>0.05)$.

Table 8. Lymphocyte (cells/ $\mu \mathrm{L}$ ) means (minimum and maximum values) of Ile de France and Santa Ines suckling lambs experimentally infected with Haemonchus contortus and non-infected control

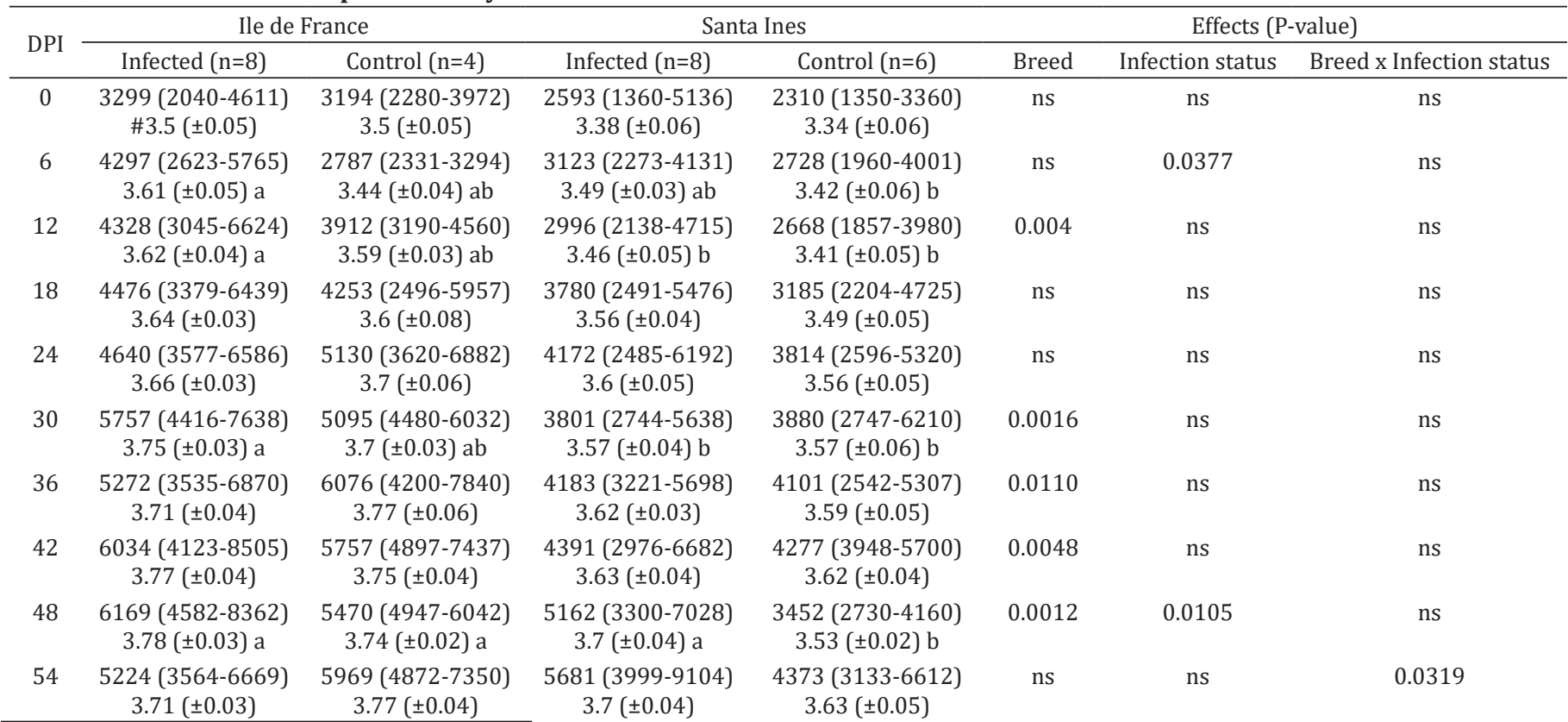

DPI = Days post first infection; \# means ( \pm standard error) of data transformed using log $(\mathrm{x}+1)$; In each row, arithmetic means with different lower case letters are significantly different by Tukey test $(P<0.05)$; ns = not significant $(P>0.05)$. 
status effect at 6 DPI and 48 DPI. Significant Breed x Infection status effect was found only at 54 DPI (68 days old). Infected Santa Ines had a higher lymphocyte count than non-infected Santa Ines lambs $(P=0.0001)$ at 48 DPI (62 days old).

With regards to monocytes means (Table 9), there was an infection status effect at 18 DPI ( 32 days old) $(P=0.0321)$. A significant Breed $\mathrm{x}$ Infection interaction, and a breed effect $(P=0.002)$ were observed at 36 DPI (50 days old). Infected Ile de France had higher number of monocytes than non-infected Ile de France lambs at 36 DPI (50 days old) $(P=0.0051)$.

\section{DISCUSSION}

Clinical signs caused by Haemonchus contortus infection, such as anaemia, apathy and submandibular oedema were absent in the Santa Ines and Ile de France suckling lambs of the present study. The number of $H$. contortus $\mathrm{L} 3$ for conducting experimental infection did not cause significant reduction in PCV, TPP and RBC means over the experiment.

According to Jain (1986), PCV and RBC for healthy sheep are $27-45 \%$, and 8-16 × 10\% $/ \mu \mathrm{L}$, respectively. Santa Ines and Ile de France suckling lambs from infected and control groups presented PCV means within reference interval for healthy sheep almost all over the trial, excepting for non-infected Santa Ines group in the first days of age. By the age of 38 days old (24 DPI), Santa Ines lambs (infected and control) presented PCV means over $30 \%$. In addition, Ile de France lambs had $\mathrm{RBC}$ counts under reference interval at 14 days old, and Santa Ines lambs from infected and non-infected groups presented RBC below reference values until 26 days of age (12 DPI).

Pereira et al. (2015) observed that healthy Santa Ines lambs under 75 days old had PVC and RBC means of $29.15 \%$ and $11.72 \times 10^{6} / \mu \mathrm{L}$, respectively. We observed that Santa Ines suckling lambs up to 68 days of age had, in some time points,
PCV means and RBC count approximated of those found by Pereira et al. (2015). In a survey conducted by Meneghini et al. (2016) healthy Santa Ines lambs up to 6 months old presented RBC and PCV means of $11.98 \times 10^{6} / \mu \mathrm{L}$ and $32.59 \%$, respectively.

Our results showed that for Santa Ines suckling lambs (infected and non-infected) between 14 and 32 days old, while RBC count increased, PCV values decreased. Considering only non-infected groups of both breeds as control reference groups, low PCV and RBC values appears to be a breed characteristic of Santa Ines lambs during the first few days of life, once both variables for Ile de France lambs remained stable.

Our results indicated that healthy Santa Ines and Ile de France suckling lambs up to 68 days old, had TPP means between 5.0 and $6.2 \mathrm{~g} / \mathrm{dL}$. These results of Ile de France and Santa Ines of both groups were below the reference intervals at least until 50 days old (36 DPI) for Ile de France lambs, and 68 days old (54 DPI) for Santa Ines lambs. Our results for non-infected Santa Ines lambs were in disagreement with those found by Pereira et al. (2015) that noted $7.09 \mathrm{~g} / \mathrm{dL}$ as TPP mean in Santa Ines lambs up to 75 days old. Healthy Manchega Spanish lambs up to 30 days of age had TPP mean of $6.4 \mathrm{~g} / \mathrm{dL}$, while in lambs aged between 30 and 70 days old, mean was 5.7g/dL (Bórnez et al. 2009).

Comparing to their respective control groups (non-infected), infected Santa Ines lambs had higher circulating WBC than infected Ile de France lambs, but such differences were not significant. In studies focusing on differences between susceptible and resistant breeds, Bowdridge et al. (2015) reported similar results in St. Croix (resistant) that had higher WBC than wool lambs (susceptible) after experimental infection with $H$. contortus.

Regarding to lymphocyte counts, infected groups presented higher means than their respective non-infected control

Table 9. Monocyte (cells $/ \mu \mathrm{L}$ ) means (minimum and maximum values) of Ile de France and Santa Ines suckling lambs experimentally infected with Haemonchus contortus and non-infected control

\begin{tabular}{|c|c|c|c|c|c|c|c|}
\hline \multirow{2}{*}{ DPI } & \multicolumn{2}{|c|}{ Ile de France } & \multicolumn{2}{|c|}{ Santa Ines } & \multicolumn{3}{|c|}{ Effects (P-value) } \\
\hline & Infected $(n=8)$ & Control $(n=4)$ & Infected $(\mathrm{n}=8)$ & Control $(n=6)$ & Breed & Infection status & Breed x Infection status \\
\hline 0 & $\begin{array}{c}28(0-87) \\
\# 0.7( \pm 0.34)\end{array}$ & $\begin{array}{l}127(0-252) \\
1.64( \pm 0.55)\end{array}$ & $\begin{array}{c}80(0-180) \\
1.47( \pm 0.33)\end{array}$ & $\begin{array}{c}73(0-160) \\
1.33( \pm 0.43)\end{array}$ & ns & ns & ns \\
\hline 6 & $\begin{array}{l}83(0-378) \\
1.1( \pm 0.41)\end{array}$ & $\begin{array}{c}55(0-108) \\
1.26( \pm 0.64)\end{array}$ & $\begin{array}{l}92(0-303) \\
1.1( \pm 0.42)\end{array}$ & $\begin{array}{l}110(0-350) \\
1.42( \pm 0.46)\end{array}$ & ns & ns & ns \\
\hline 12 & $\begin{array}{c}91(0-210) \\
1.52( \pm 0.34)\end{array}$ & $\begin{array}{c}96(0-152) \\
1.57( \pm 0.53)\end{array}$ & $\begin{array}{c}89(0-208) \\
1.53( \pm 0.34)\end{array}$ & $\begin{array}{c}63(0-199) \\
1.03( \pm 0.46)\end{array}$ & ns & ns & ns \\
\hline 18 & $\begin{array}{l}148(0-344) \\
1.87( \pm 0.29)\end{array}$ & $\begin{array}{c}57(0-122) \\
1.03( \pm 0.59)\end{array}$ & $\begin{array}{c}74(0-239) \\
1.43( \pm 0.32)\end{array}$ & $\begin{array}{l}41(0-127) \\
0.7( \pm 0.44)\end{array}$ & ns & 0.0321 & ns \\
\hline 24 & $\begin{array}{l}102(0-225) \\
1.76( \pm 0.26)\end{array}$ & $\begin{array}{c}185(0-333) \\
1.79( \pm 0.6)\end{array}$ & $\begin{array}{c}61(0-124) \\
1.24( \pm 0.36)\end{array}$ & $\begin{array}{c}49(0-70) \\
1.48( \pm 0.3)\end{array}$ & ns & ns & ns \\
\hline 30 & $\begin{array}{c}74(0-173) \\
1.48( \pm 0.33)\end{array}$ & $\begin{array}{l}151(0-416) \\
1.22( \pm 0.71)\end{array}$ & $\begin{array}{c}89(0-276) \\
1.47( \pm 0.34)\end{array}$ & $\begin{array}{c}92(0-201) \\
1.41( \pm 0.45)\end{array}$ & ns & ns & ns \\
\hline 36 & $\begin{array}{c}128(0-336) \\
1.61( \pm 0.36) \mathrm{a}\end{array}$ & $\begin{array}{c}0(0-0) \\
0( \pm 0.0) b\end{array}$ & $\begin{array}{c}108(0-201) \\
1.79( \pm 0.27) \mathrm{a}\end{array}$ & $\begin{array}{l}136(74-261) \\
2.09( \pm 0.08) \mathrm{a}\end{array}$ & 0.002 & ns & 0.0071 \\
\hline 42 & $\begin{array}{l}54(0-363) \\
0.55( \pm 36)\end{array}$ & $\begin{array}{l}121(0-219) \\
1.64( \pm 0.55)\end{array}$ & $\begin{array}{l}129(0-359) \\
1.82( \pm 0.28)\end{array}$ & $\begin{array}{c}78(0-201) \\
1.36( \pm 0.43)\end{array}$ & ns & ns & ns \\
\hline 48 & $\begin{array}{l}152(0-865) \\
1.34( \pm 0.41)\end{array}$ & $\begin{array}{c}70(0-194) \\
1.06( \pm 0.55)\end{array}$ & $\begin{array}{c}254(126-468) \\
2.36( \pm 0.07)\end{array}$ & $\begin{array}{l}108(0-239) \\
1.71( \pm 0.35)\end{array}$ & ns & ns & ns \\
\hline 54 & $\begin{array}{c}91(0-247) \\
1.51( \pm 0.34)\end{array}$ & $\begin{array}{c}23(0-94) \\
1.06( \pm 0.61)\end{array}$ & $\begin{array}{l}68(0-227) \\
1.05( \pm 0.4)\end{array}$ & $\begin{array}{c}45(0-97) \\
0.98( \pm 0.44)\end{array}$ & ns & ns & ns \\
\hline
\end{tabular}

DPI = Days post first infection; \# means ( \pm standard error) of data transformed using log $(\mathrm{x}+1)$; In each row, arithmetic means with different lower case letters are significantly different by Tukey test $(P<0.05)$; ns $=$ not significant $(P>0.05)$. 
groups, however, infected Santa Ines lambs had significantly higher number of cells than non-infected Santa Ines only at 62 days old (48 DPI). Increases in lymphocyte number and WBC count were related to the recruitment of defence Th2 cells to the parasite-host interaction site (Balic et al. 2000, Bowdridge et al. 2015).

Significant differences in blood eosinophil count between Santa Ines groups (infected and uninfected control) were observed in two time points. Comparing infected groups only, there was a significant difference between breeds in circulating eosinophil count at 54 DPI (68 days old), with infected Santa Ines lambs presenting the highest mean. However, the number of eosinophils was within the reference interval for sheep in both breeds. Rocha et al. (2005) in study with Santa Ines and Ile the France suckling lambs naturally infected by gastrointestinal nematodes, observed no significant differences between breeds regarding to eosinophil counts, although Santa Ines lambs had higher means of eosinophils than Ile the France lambs over the trial. In a $H$. contortus chronic infection, naturally infected Santa Ines lambs (8 months old) had higher mean blood eosinophil counts than Ile de France lambs (Albuquerque et al. 2019).

Eosinophils are important in the defence against infective larvae of gastrointestinal nematodes (Meeusen \& Balic 2000, Tizard 2014), and eosinophilia is reported as a protective response against $H$. contortus (Terefe et al. 2005). Additionally, they have a variety of receptors (including those for cytokines, and immunoglobulins) that enable them to be recruited into affected tissues (Makepeace et al. 2012). In vitro studies have shown that eosinophils are able of reducing $H$. contortus L3 motility, and decrease infectivity of L3 in the host (Terefe et al. 2005, Holt et al. 2015). On tissue, eosinophils can limit parasite growth, decrease fecundity of the parasite, and also damaging it morphologically (Balic et al. 2000, McRae et al. 2015, Huang \& Appleton 2016).

A type 2 immune (Th2) response plays a major role in host protection against gastrointestinal nematodes (GIN) infections, through the induction of cytokines, antibody production, and recruitment of multiple inflammatory cells (McRae et al. 2015, Sorobetea et al. 2018). After GIN getting the site of infection, their antigens are presented by antigenpresenting cells to naïve T cells, that result in the release of Th2-associated cytokines (Makepeace et al. 2012, McRae et al. 2015). Interleukins secreted by Th2 cells, such as interleukin (IL) 5 and IL-13, can stimulate stem cell of bone morrow into eosinophil differentiation (Park \& Bochner 2010), and to their recruitment to the site of the infection. For this reason, eosinophil number increases significantly in the blood (Meeusen et al. 2005, Tizard 2014) and after getting into circulation, eosinophils rapidly migrate into tissues to the site of infection (Anthony et al. 2007). Resistant sheep breeds may present a higher production of interleukins related to Th2 response, especially IL-4, IL-5 and IL-13 (Terefe et al. 2007).

Several studies have shown that Santa Ines sheep is considered more resistant to GIN infections compared to Ile de France sheep at different age categories (Amarante et al. 2004,2009 , Rocha et al.2005, 2011), mainly because of Santa Ines sheep present an earlier and robust initial Th2 response (Albuquerque et al. 2019).

Absence of difference between susceptible and resistant sheep breeds in circulating eosinophil count may thus reflect immune responses to different stages of GIN infection (Bowdridge et al. 2015). As supposed by Albuquerque et al. (2019), our results suggest that Santa Ines lambs had an increase in eosinophil production, however differences on circulation number were not identified, because they also promote a rapid migration of such cells to the site of parasite infection.

Neuthrophil and monocyte counts were similar in both breeds. Different results were reported in older lambs, previously infected and then artificially challenged with $H$. contortus. In this study, St. Croix hair (resistant) had higher WBC and neutrophil counts than wool lambs (susceptible) (Bowdridge et al. 2015). In vitro, neutrophils from these resistant St. Croix lambs, bound to $H$. contortus $\mathrm{L} 3$ after reinfection, in a higher percentage than neutrophils from susceptible Suffolk lambs (Garza et al. 2018). Neutrophils are important cells that guide and support the innate and adaptive immune response (Mantovani et al. 2011). Such cells secrete a variety of proinflammatory mediators that amplify the inflammatory process, and their counts indicate an important role in modulating immune response (Bowdridge et al. 2015, Weiss \& Wardrop 2010) and as indicator of effective host response against helminthiasis (Ortolani et al. 2013). After a brief period in circulation, neutrophils may transmigrate into site of parasitic helminth invasion, and working in coordination with other cell populations, including eosinophils and macrophages, prevent larval establishment through damage tissue-dwelling helminths (Anthony et al. 2007).

Even within the reference range for sheep and without statistical differences, infected Santa Ines lambs presented highest means of basophils, excepting at 30 DPI (44 days old). Such increased in basophils number coincided with the increase in eosinophils values at 20 days old (6 DPI) as well. In accordance with our results, Bowdridge et al. (2015) observed that St. Croix hair (resistant) presented higher means of basophils than wool (susceptible) lambs. Basophils are relatively rare and short-lived cell type, however they play an important role in amplifying type 2 immune response to helminth infections through the production in large quantities of effector molecules including histamine, cytokines, chemokines, and lipid mediators (Mitre \& Nutman 2006, Eberle \& Voehringer 2016).

\section{CONCLUSION}

The mild Haemonchus cortortus infection did not induce significant changes in the blood variables of the Ile de France and Santa Ines suckling lambs, although infected Santa Ines lambs had higher counts of eosinophils. Healthy Santa Ines and Ile de France suckling lambs up to 68 days old, had total plasma protein (TPP) means between 5.0 and $6.2 \mathrm{~g} / \mathrm{dL}$. Low packed cell volume (PCV) and red blood cell count (RBC) count could be a breed characteristic of Santa Ines lambs during the first days of life.

Acknowledgements.- The authors would like to thank Serginara D. Rodrigues for the technical support. José Gabriel G. Lins received scholarship from "Coordenação de Aperfeiçoamento de Pessoal de Nível Superior" (CAPES), Brazil; Fabiana A. Almeida received a scholarship from "Fundação de Amparo à Pesquisa do Estado de São Paulo” (FAPESP), grant \#2015/25718-7; and Alessandro F. T. Amarante is in receipt of a fellowship from "Conselho Nacional de Desenvolvimento Científico e Tecnológico” (CNPq) (grant\#305187/2017-1). 
Conflicts of interest statement.- The authors declare no conflicts of interest regarding the publication of this paper.

\section{REFERENCES}

Albuquerque A.C.A., Bassetto C.C., Almeida F.A., Hildersley K.A., McNeilly T.N., Britton C. \& Amarante A.F.T. 2019. Differences in immune responses to Haemonchus contortus infection in the susceptible Ile de France and the resistant Santa Ines sheep under different anthelmintic treatments regimens. Vet. Res. 50:104. <https://dx.doi.org/10.1186/s13567-019-0722-3>

Almeida F.A., Garcia K.C.O.D., Torgerson P.R. \& Amarante A.F.T. 2010. Multiple resistance to anthelmintics by Haemonchus contortus and Trichostrongylus colubriformis in sheep in Brazil. Parasitol. Int. 59(4):622-625. <https:// dx.doi.org/10.1016/j.parint.2010.09.006><PMid:20887800>

Amarante A.F.T. \& Amarante M.R.V. 2016. Advances in the diagnosis of the gastrointestinal nematode infections in ruminants. Braz. J. Vet. Res. Anim. Sci. 53(2):127-137.<https://dx.doi.org/10.11606/issn.1678-4456. v53i2p127-137>

Amarante A.F.T. 2014. Sustainable worm control practices in South America. Small Rumin. Res. 118(1/3):56-62. <https://dx.doi.org/10.1016/j. smallrumres.2013.12.016>

Amarante A.F.T., Bricarello P.A., Rocha R.A. \& Gennari S.M. 2004. Resistance of Santa Ines, Suffolk and Ile de France sheep to naturally acquires gastrointestinal nematode infections. Vet. Parasitol. 120(1/2):91-106. <https://dx.doi.org/10.1016/j.vetpar.2003.12.004>

Amarante A.F.T., Susin I., Rocha R.A., Silva M.B., Mendes C.Q. \& Pires A.V. 2009. Resistance of Santa Ines and crossbred ewes to naturally acquired gastrointestinal nematode infections. Vet. Parasitol. 165(3/4):273-280. <https://dx.doi.org/10.1016/j.vetpar.2009.07.009>

Anthony R.M., Rutizky L.I., Urban Jr J.F., Stadecker M.J. \& Gause W.C. 2007. Protective immune mechanisms in helminth infection. Nat. Rev. Immunol. 7(12):975-987. <https://dx.doi.org/10.1038/nri2199><PMid:18007680>

Bahirathan M., Miller J.E., Barras S.R. \& Kearney M.T. 1996. Susceptibility of Suffolk and Gulf Coast Native suckling lambs to naturally acquired strongylate infection. Vet. Parasitol. 65(3/4):259-268. <https://dx.doi org/10.1016/s0304-4017(96)00969-7><PMid:8983151>

Balic A., Bowles V.M. \& Meeusen E.N.T. 2000. The immunobiology of gastrointestinal nematode infections in ruminants. Adv. Parasit. 45:181-241. <https://dx.doi.org/10.1016/s0065-308x(00)45005-0><PMid:10751941>

Bórnez R., Linares M.B. \& Vergara H. 2009. Haematological, hormonal and biochemical blood parameters in lamb: effect of age and blood sampling time. Livest. Sci. 121(2/3):200-206. <https://dx.doi.org/10.1016/j. livsci.2008.06.009>

Bowdridge S.A., Zajac A.M. \& Notter D.R. 2015. St Croix sheep produce a rapid and greater cellular immune response contributing to reduce establishment of Haemonchus contortus. Vet. Parasitol. 208(3/4):204-210. <https://dx.doi.org/10.1016/j.vetpar.2015.01.019><PMid:25698414>

Eberle J.U. \& Voehringer D. 2016. Role of basophils in protective immunity to parasitic infections. Semin. Immunopathol. 38(5):605-613. <https://dx.doi.org/10.1007/s00281-016-0563-3> <PMid:27116557>

Echevarria F.A.M., Armour J. \& Duncan J.L. 1991. Efficacy of some anthelmintics on a ivermectin-resistant strain of Haemonchus contortus in sheep. Vet. Parasitol, 39(3/4):279-384. <https://dx.doi.org/10.1016/03044017(91)90044-V>

Emery D.L., Hunt P.W. \& Le Jambre L.F. 2016. Haemonchus contortus: the then and now, and where to from here? Int. J. Parasit. 46(12):755-769. <https://dx.doi.org/10.1016/j.ijpara.2016.07.001><PMid:27620133>

Garza J.J., Greiner S.P. \& Bowdridge S.A. 2018. Ovine vital neutrophil extracellular traps bind and impair Haemonchus contortus L3 in a breed-dependent manner. Parasite Immunol. 40(9):e12572.<https://dx.doi.org/10.1111/ pim.12572><PMid:29992577>
Holt R.M., Shepherd E.A., Ammer A.G. \& Bowdridge S.A. 2015. Effects of peripheral blood mononuclear cells on Haemonchus contortus larval motility in vitro. Parasite Immunol. 37(10):553-556. <https://dx.doi. org/10.1111/pim.12219><PMid:26223339>

Huang L. \& Appleton J.A. 2016. Eosinophils in helminth infection: defenders and dupes. Trends Parasitol. 32(10):798-807.<https://dx.doi.org/10.1016/j. pt.2016.05.004><PMid:27262918>

Jain N.C. 1986. Schalm's Veterinary Haematology. 4th ed. Lea and Febiger, Philadelphia. 1221p.

Kumar S., Jakhar K.K., Singh S., Potliya S., Kumar K. \& Pal M. 2015. Clinicopathological studies of gastrointestinal tract disorders in sheep with parasitic infection. Vet. World 8(1):29-32. <https://dx.doi.org/10.14202/ vetworld.2015.29-32><PMid:27046991>

Makepeace B.L., Martin C., Turner J.D. \& Specht S. 2012. Granulocytes in helminth infection - who is calling the shots? Curr. Med. Chem. 19(10):1567-1586. <https://dx.doi.org/10.2174/092986712799828337><PMid:22360486>

Mantovani A., Cassatella M.A., Costantini C. \& Jaillon S. 2011. Neutrophils in the activation and regulation of innate and adaptive immunity. Nat. Rev. Immunol. 11(8):519-531. <https://dx.doi.org/10.1038/nri3024> $<$ PMid:21785456>

McRae K.M., Stear M.J., Good B. \& Keane O.M. 2015. The host immune response to gastrointestinal nematode infection in sheep. Parasite Immunol. 37(12):605-613. <https://dx.doi.org/10.1111/pim.12290> <PMid:26480845>

Meeusen E.N. \& Balic A. 2000. Do eosinophils have a role in the killing of helminth parasites? Parasitol. Today 16(3):95-101. <https://dx.doi. org/10.1016/s0169-4758(99)01607-5> <PMid:10689327>

Meeusen E.N.T., Balic A. \& Bowles V.M. 2005. Cells, cytokines and other molecules associated with rejection of gastrointestinal nematode parasites. Vet. Immunol. Immunop. 108(1/2):21-125.<https://dx.doi.org/10.1016/j. vetimm.2005.07.002><PMid:16099054>

Meneghini R.C.M., Benesi F.J., Henriques L.C.S., Rizzo H., Meira Junior E.B.S. \& Gregory L. 2016. Hemogram of healthy sheep (Ovisaries) of the Santa Ines breed raised in the region of Piedade, São Paulo State: influence of age and sex. Braz. J. Vet. Res. Anim. Sci. 53(4):1-7. <https://dx.doi.org/10.11606/ issn.1678-4456.bjvras.2016.79665>

Mitre E. \& Nutman T.B. 2006. Basophils, basophilia and helminth infections. Chem. Immunol. Allergy 90:141-156. <https://dx.doi.org/10.1159/000088886> <PMid:16210908>

Ortolani E.L., Leal M.L.R., Minervino H.H., Aires A.R., Coop R.L., Jackson F. \& Suttle N.F. 2013. Effects of parasitism on cellular immune response in sheep experimentally infected with Haemonchus contortus. Vet. Parasitol. 196(1/2):230-234. <https://dx.doi.org/10.1016/j.vetpar.2013.02.014> <PMid:23522899>

Park Y.M. \& Bochner B.S. 2010. Eosinophil survival and apoptosis in health and disease. Allergy Asthma Immunol. Res. 2(2):87-101.<https://dx.doi. org/10.4168/aair.2010.2.2.87><PMid:20358022>

Pereira F.B., Bezerra L.R., Marques C.A.T., Araújo M.J., Torreão J.N.C. \& Machado L.P. 2015. Perfil hematológico de ovelhas Santa Inês suplementadas a pasto no terço final de gestação e no pós-parto. Ciênc. Anim. Bras. 16(3):350-357. <https://dx.doi.org/10.1590/1089-6891v16i327573>

Rocha R.A., Amarante A.F.T. \& Bricarello P.A. 2005. Resistance of Santa Inês and Ile de France suckling lambs to gastrointestinal nematode infections. Revta Bras. Parasitol. Vet. 14(1):17-20. <PMid:16153339>

Rocha R.A., Bricarello P.A., Silva M.B., Houdijk J.G.M., Almeida F.A., Cardia D.F.F., Amarante A.F.T. 2011. Influence of protein supplementation during late pregnancy and lactation on the resistance of Santa Ines and Ile de France ewes to Haemonchus contortus. Vet. Parasitol. 181(2/4):229-238. <https://dx.doi.org/10.1016/j.vetpar.2011.03.055><PMid:21726941>

Shakya K.P., Miller J.E. \& Horobov D.W. 2009. A Th2 type of imumune response is associated with increased resistance to Haemonchus contortus in 
naturally infected Gulf Coast Native lambs. Vet. Parasitol. 163(1/2):57-66. <https://dx.doi.org/10.1016/j.vetpar.2009.03.052 > <PMid:19406579>

Sorobetea D., Svensson-Frej M., Grencis R. 2018. Immunity to gastrointestinal nematode infections. Mucosal Immunol. 11(2):304-315. <https://dx.doi. org/10.1038/mi.2017.113><PMid:29297502>

Terefe G., Lacroux C., Andreoletti O., Grisez C., Prevot F., Bergeaud J.P., Penicaud J., Rouillon V., Gruner L., Brunel J.C., Francois D., Bouix J., Dorchies P. \& Jacquiet P. 2007. Immune response to Haemonchus contortus infection in susceptible (INRA 401) and resistant (Barbados Black Belly) breeds of lambs. Parasite Immunol. 29(8):415-424. <https://dx.doi. org/10.1111/j.1365-3024.2007.00958.x ><PMid:17650183>

Terefe G., Yacob H.T., Grisez C., Prevot F., Dumas E., Bergeaud J.P., Dorchies P., Hoste H. \& Jacquiet P. 2005. Haemonchus contortus egg excretion and female length reduction in sheep previously infected with Oestrus ovis (Diptera: Oestridae) larvae. Vet. Parasitol. 128(3/4):271-283. <https:// dx.doi.org/10.1016/j.vetpar.2004.11.036><PMid:15740864>

Tizard I.R. 2014. Imunologia Veterinária. 9ae ed. Elsevier, Rio de Janeiro. 568p.

Ueno H. \& Gonçalves P.C. 1998. Manual para diagnóstico das helmintoses de ruminantes. $4^{\mathrm{a}}$ ed. International Cooperation Agency, Japan. 166p.

Weiser G. 2012. Laboratory technology for veterinary medicine, p.3-36. In: Thrall M.A. (Ed.), Veterinary Hematology and Clinical Chemistry. $2^{\underline{a}}$ ed. Wiley-Blackwell, Iowa.

Weiss D.J. \& Wardrop K.J. 2010. Schalm's Veterinary Hemathology. 6 ${ }^{\mathrm{a}}$ ed. Wiley-Blackwell, Iowa. 1232p. 